\title{
Three-dimensional anatomical accuracy of cranial models created by rapid prototyping techniques validated using a neuronavigation station
}

Type: Article

Abstract:

In neurosurgery and ear, nose and throat surgery the application of computerised navigation systems for guiding operations has been expanding rapidly. However, suitable models to train surgeons in using navigation systems are not yet available. We have developed a technique using an industrial, rapid prototyping process from which accurate spatial models of the cranium, its contents and pathology can be reproduced for teaching. We were able to register, validate and navigate using these models with common available navigation systems such as the Medtronic StealthStation S7 (R).

\begin{tabular}{|c|c|}
\hline Author & $\begin{array}{l}\text { - Waran, V. } \\
\text { - } \text { Devaraj, P. } \\
\text { - } \text { Muthusan, T. H. } \\
\text { - Rathinam, K. A. K. } \\
\text { - Balakrishnan, Y. K. } \\
\text { - Tung, T. S. } \\
\text { - Raman, R. } \\
\text { - Rahman, Z. A. A. }\end{array}$ \\
\hline Source & Journal of Clinical Neuroscience \\
\hline ISSN & 0967-5868 \\
\hline DOI & 10.1016/j.jocn.2011.07.031 \\
\hline Volume (Issue) & $19(4)$ \\
\hline Page & $574-577$ \\
\hline Year & 2012 \\
\hline
\end{tabular}

Keyword:

Neuronavigation, Rapid prototyping technique, Surgical heads, temporal bone, surgery, stereolithography, technology, fractures, spine

Please Cite As:

WARAN, V., DEVARAJ, P., CHANDRAN, T. H., MUTHUSAMY, K. A., RATHINAM, A. K., BALAKRISHNAN, Y. K., TUNG, T. S., RAMAN, R. \& RAHMAN, Z. A. A. 2012. Three-dimensional anatomical accuracy of cranial models created by rapid prototyping techniques validated using a neuronavigation station. Journal of Clinical Neuroscience, 19, 574-577. 
URL:

- apps.webofknowledge.com Search by Accession No: 000301805700019

- http://www.scopus.com/inward/record.url?eid=2-s2.084857787121\&partner|D=40\&md5=9d0084b9b94a8a3b8460ff4e0932ad78

- $\quad$ http://www.ncbi.nlm.nih.gov/pubmed/22305869

- http://www.sciencedirect.com/science/article/pii/S0967586811005364 